NOTES

\title{
Existence of Concanavaline A Type $\beta$-Sheet Structure Shown by Reduction of Disulfide Bonds of a Dimeric Globular Protein
}

\author{
Tomoko KomIYAMA and Makoto MIWA \\ Department of Industrial Chemistry, Seikei University, \\ Musashino, Tokyo 180, Japan
}

(Received September 18, 1984)

\begin{abstract}
KEY WORDS Subunit Interface / Disulfide Bonds / Reductive Cleavage / Secondary Structure / Fluorescence / CD /
\end{abstract}

Streptomyces subtilisin inhibitor (SSI) is a stable dimeric globular protein comprised of two identical subunits. Figure 1 represents a schematic drawing of the SSI subunit secondary structure. The subunit contains $113^{\prime}$ residues and has a molecular weight of 11500 . XRay crystallographic study indicated the interface between subunits to be supported by the $\beta$-sheet interaction, which is analogous to concanavaline $\mathrm{A}^{1}$. A dimer of concanavaline A comes into contact with another dimer through a $\beta$-sheet $-\beta$-sheet interaction. ${ }^{2}$

The hydrophobic core of the SSI dimer consists of a central $\beta$-sheet with peripheral $\alpha$ helices and disulfide bonds as shown in Figure 1. ${ }^{1}$ Cys 50 and Cys 101 in two disulfide bonds are located in the $\alpha_{1}$-helix and $\alpha_{2}$-helix, respectively. The first disulfide bond (Cys 35Cys 50) connects the $\alpha_{1}$-helix to the $\beta_{2}$-strand. The latter bond connects the $\alpha_{2}$-helix to the rimming peptide chain to form a loop containing the reactive site (Met 73-Val 74) and $\beta_{4}$ and $\beta_{5}$ strands. Considering the disulfide bonds to be important in the SSI structure, we examined the effect of a reducing agent on the secondary structure of SSI and found a sharp change in the profile of the CD spectrum. The far-UV CD spectrum of the fully. reduced SSI was similar to that of concana- valine $A$, indicating the subunit interface of SSI to be the concanavaline A type $\beta$-sheet structure.

\section{EXPERIMENTAL}

SSI, kindly provided by Prof. S. Murao and his coworkers of University of Osaka Prefecture, was purified by an ordinary method. ${ }^{3}$ Concanavaline A was purchased from

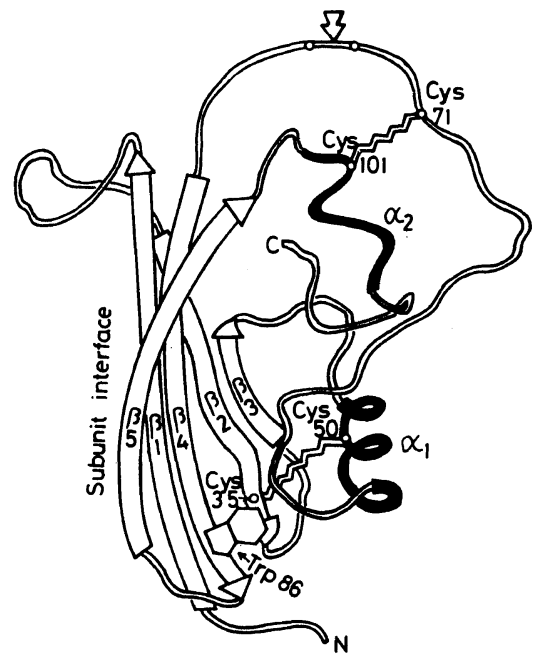

Figure 1. Schematic diagram drawn on the basis of the $\mathrm{X}$-ray study. ${ }^{1}$ The arrow at the top indicates the scissile bond. Important residues and peptide chains are illustrated in the figure. 
Fluka and used without further purification. Dithioerythritol (DTE) from Tokyo Kasei Kogyo Co., was used as received. $N$-(1Anilinonaphthyl-4-)-maleimide (ANM) and $N$-acetyl-L-cysteine ( $N$-acetyl-L-Cys) were purchased from Wako Pure Chemical Ind., and Takara Kohsan Co., respectively.

The reduced SSI was prepared in vacuo as follows. An SSI solution $\left(0.3 \mathrm{mg} \mathrm{ml}^{-1}\right)$ in $0.05 \mathrm{M}$ phosphate buffer $(\mathrm{pH} 7.0, \mu=0.1$ $\mathrm{NaCl}$ ) was deaerated by cycles of pumping and thawing. Forty times (in SSI dimer basis) excess solid DTE was added to the deaerated SSI solution in vacuo by breaking the seal between the SSI solution and DTE. All procedures were carried out in a vacuum system containing the reactive vessel and optical cells. The cells into which the reduced SSI solution was transferred were sealed off for spectra measurements.

The amounts of thiol groups produced from reduction were quantitatively analyzed by labeling them with ANM. This reagent itself is non-fluorescent, but ANM complexed with thiol groups shows intense fluorescence at about $460 \mathrm{~nm}^{4}$ The complex of this reagent with thiol groups due to excess DTE was removed by dialysis. We used Spectra/Por 1 dialysis tubing (Spectrum Medical Industrial Inc., Los Angeles) whose cutoff molecular weight was $6000-8000$. The concentration of thiol group attributable to the reduced protein was determined by comparing the fluorescence intensity obtained following dialysis with that of the authentic sample, an equimolar mixture of ANM and $N$-acetyl-LCys.

CD spectra were measured with a JASCO J40 circular dichroism spectrophotometer at $20^{\circ} \mathrm{C}$. The optical length of cells was $1 \mathrm{~mm}$. Fluorescence spectra were measured with a Hitachi 650 spectrofluorometer.

\section{RESULTS AND DISCUSSION}

Figure 2 shows the far-UV CD spectrum of

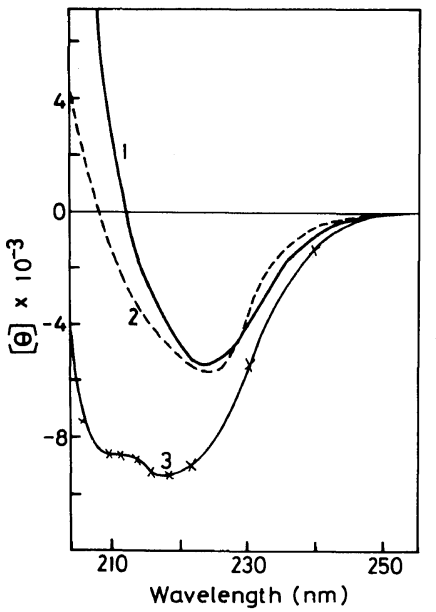

Figure 2. $C D$ spectra of the reduced SSI (curve 1), native concanavaline A (curve 2) and native SSI (curve 3 ). Tow disulfide bonds were reduced by forty times excess DTE at $20^{\circ} \mathrm{C}$ in vacuo. Fitting plots $(\times)$ are shown on curve 3 .

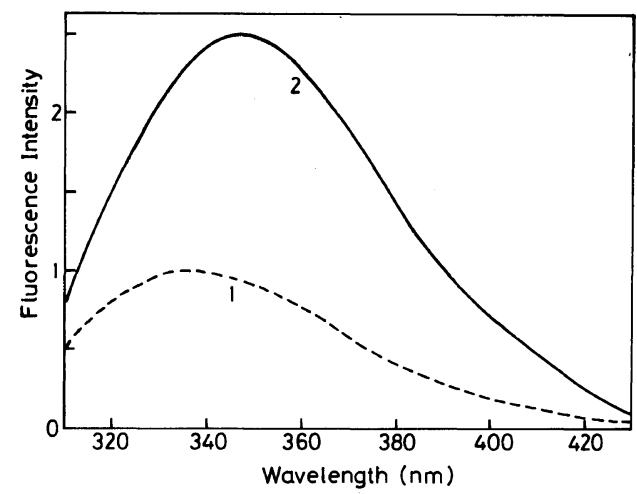

Figure 3. Fluorescence spectra of a $\operatorname{Trp}$ residue at $\mathrm{pH}$ 7 and $20^{\circ} \mathrm{C}$ of the native SSI (curve 1) and SSI solution treated with forty times excess DTE in vacuo (curve 2).

SSI the solution treated with forty times excess DTE for $20 \mathrm{~h}$ in vacuo. Since reduction of disulfide bonds of SSI proceeds slowly at room temperature, sealing the solution in vacuo was necessary to prevent re-oxidization. The CD spectrum of the native SSI is also shown in Figure 2 for comparison. A sharp change was observed for the reduced solution. This change was not seen without DTE. Fluorescence intensity of ANM complexed with thiol groups of the reduced SSI was eight times that of 
ANM complexed with one thiol group of $N$ acetyl-L-Cys, indicating all disulfides bonds of SSI to be completely broken. The reliability of this quantitative analysis of thiol groups was checked by repeating the procedure three times. Experimental error was $9 \%$. A remarkable change was induced in the intrinsic fluorescence of a Trp residue by reduction, as shown in Figure 3. A single Trp residue is buried in the hydrophobic core ${ }^{1}$ (Figure 1) and emits weak fluorescence in the native state..$^{5-7}$ $\mathrm{X}$-Ray studies have shown the sulfur atom of Cys 35 to be in contact with the benzene ring of Trp 86. ${ }^{1,8}$ Reduction of a disulfide bond of Cys 35-Cys 50 makes the Trp residue free from the sulfur atom as a fluorescence quencher and enhances the fluorescence intensity.

We noticed an unexpected homology between the CD spectrum of the reduced SSI and that of the native concanavaline $\mathrm{A}$ as shown in Figure 2. The reduction of two disulfide bonds unfolded two $\alpha$-helices joined to disulfide bonds as mentioned in the introduction (Figure 1), and melted the helical component of CD. It is clear that concanavaline A type $\mathrm{CD}$ pattern in the $\mathrm{CD}$ of native SSI emerges by reduction of the disulfide bonds.

Concanavaline $\mathrm{A}$ is a typical $\beta$-protein to which ordinary $\mathrm{CD}$ analyses are not applicable. ${ }^{9,10}$ In ordinary $C D$ analyses, a fixed analysis function is used for each secondary structure. ${ }^{9,11}$ The CD spectrum of native SSI cannot be analyzed by ordinary methods which gave inconsistent plots with the experimental CD spectrum of native SSI. ${ }^{12}$ The CD spectrum of SSI was analyzed by a new method developed by Provencher and Glöckner ${ }^{13}$ and applicable to concanavaline A. Fractional contents of the secondary structure of SSI were $24 \% \alpha$-helix, $36 \% \beta$-sheet and $40 \%$ remainder. ${ }^{14}$ The class remainder contained $\beta$-turn, random coils and nonpeptidic chromophores. The contents of secondary structure calculated as above was at variance with the results obtained from X-ray crystallography, ${ }^{1}$ although fitting plots were consistent with the
Table I. Secondary structure composition of SSI determined by various ways Upper part shows the fractional content $(f)$ of each secondary structure. In the lower part, $\alpha$-helix and $\beta$-sheet polypeptide chain segments are represented by sequence numbers.

\begin{tabular}{lcccc}
\hline & CD $^{14}$ & X-Ray & Prediction $^{15}$ & $\begin{array}{c}\text { Torsion } \\
\text { angles }^{16}\end{array}$ \\
\hline$f_{\alpha}$ & 0.24 & 0.12 & 0.23 & 0.15 \\
$f_{\beta}$ & 0.36 & 0.21 & 0.34 & 0.31 \\
$f_{\mathrm{R}}$ & 0.40 & 0.67 & 0.43 & 0.54 \\
\hline$\alpha$-Helix & & & $20-28$ & \\
region & & $47-56$ & $48-56$ & $46-55$ \\
& & $100-106$ & $99-103$ & $100-106$ \\
$\beta$-Sheet & & $9-19$ & $10-16$ & $10-17$ \\
region & & $29-35$ & $29-35$ & $29-38$ \\
& & $40-42$ & & \\
& & & $68-75$ & $71-76$ \\
& & $77-87$ & $77-87$ & $81-86$ \\
& & $89-96$ & $93-96$ & $91-95$ \\
\hline
\end{tabular}

measured CD, as shown in Figure 2.

To explain the discrepancy between X-ray and CD studies, we applied the Chou-Fasman prediction method ${ }^{15}$ and the torsion angles method. ${ }^{16}$ On the basis of a comparison of the observed values of peptide backbone torsion angles ${ }^{16}$ with the standard values, ${ }^{17}$ we assigned 15 and $31 \%$ of the total residues to $\alpha$-helix and $\beta$-sheet, respectively. The results are summarized in Table I. The $\beta$-sheet content as estimated from $\mathrm{CD}$ analysis was consistent with the values obtained by the torsion angles and prediction methods. Since X-ray data are based on the existence of hydrogen bonds, the $\beta$-sheet content may have been underestimated. By CD and the prediction the $\alpha$-helix content was overestimated. Though CD analysis and the prediction are based on information obtained from X-ray studies, ${ }^{13,15}$ the secondary structure of SSI does not belong to the conventional pattern deduced from various proteins whose structures have been determined by X-ray crystallographies. The discrep- 
ancy between $\mathrm{CD}$ and X-ray analyses thus arises from an overestimation of the $\alpha$-helix content and may possibly be associated with the fact that both helices are connected to another part of SSI by disulfide bonds. Analysis of the CD spectra of dimeric proteins should clarify the theoretical aspects of this matter.

In conclusion, two disulfides bonds of SSI subunit support two helices and the complete reduction of these bonds changes the $\mathrm{CD}$ profile of SSI to the pure $\beta$-sheet type from the mixed type of the $\alpha$-helix and $\beta$-sheet in the native state.

Acknowledgements. We thank Prof. T. Imai and Mr. T. Okamura of Seikei University for the use of the spectrofluorometer.

\section{REFERENCES}

1. Y. Mitsui, Y. Satow, Y. Watanabe, S. Hirono, and Y. Iitaka, J. Mol. Biol., 131, 697 (1979).

2. G. N. Reeke, Jr., J. W. Becker, and G. M. Edelman, J. Biol. Chem., 259, 1525 (1975).
3. S. Sato and S. Murao, Agric. Biol. Chem., 37, 1067 (1973).

4. Y. Kanaoka, M. Machida, and T. Sekine, Biochem. Biophys. Acta, 317, 563 (1973).

5. Y. Uehara, B. Tonomura, and K. Hiromi, Biochim. Biophys. Acta, 453, 513 (1976).

6. T. Komiyama, M. Miwa, S. Sato, and S. Murao, Biochim. Biophys. Acta, 493, 188 (1977).

7. T. Komiyama and M. Miwa, J. Biochem., 87, 1029 (1980).

8. Y. Satow, Y. Watanabe, and Y. Mitsui, J. Biochem., 88, 1739 (1980).

9. Y.-H. Chen, J. T. Yang, and K. H. Chau, Biochemistry, 13, 3350 (1974).

10. S. Brahms and J. Brahms, J. Möl. Biol., 138, 149 (1980).

11. C. T. Chen, C. C. Wu, and J. T. Yang, Anal. Biochem., 91, 000 (1978).

12. T. Komiyama, M. Miwa, H. Ikeda, and T. Yatabe, Abstracts, 32nd Conference on Protein Structure, Sendai, September, 1981.

13. S. W. Provencher and J. Glockner, Biochemistry, 20, 33 (1981).

14. T. Komiyama, M. Miwa, T. Yatabe, and H. Ikeda, $J$. Biochem., 95, 1569 (1984).

15. P. Y. Chou and G. D. Fasman, Ann. Rev. Biochem., 47, 251 (1978).

16. Y. Mitsui, private communication.

17. IUPAC-IUB Commision of Biochemical Nomenclature, J. Mol. Biol., 52, 1 (1970). 